Diabetes insipidus has been reported as a rare manifestation of congenital neurosyphylis, but no previous reports of water intoxication were found in the literature. While in both conditions polydipsia and polyuria occur, in diabetes insipidus urinary osmolality is well below plasma osmolality, and serum urea and sodium tend to be raised. In water intoxication, both plasma and urinary osmolality are low and serum urea and sodium are below reference values. The syndrome of inappropriate antidiuretic hormone secretion in which hyponatraemia occurs can be excluded by the absence of raised urinary osmolality. This patient has been treated with trifluoperazine, and although psychotic symptoms were effectively treated the water intake remained excessive. Dopamine has been shown to inhibit vasoprotein release in man (Lightman \& Forsling, 1980) and it is suggested that in this case its antagonism by trifluoperazine could have led to increased ADH. Demeclocycline, an antidiuretic hormone antagonist, was therefore added to the treatment, and this was followed by the return of serum electrolytes to reference values. However, the effects of neuroleptics on ADH secretion are inconsistent and I suggest that the personal habits of the patient such as smoking and tea and alcohol intake were not considered. This patient drank large quantities of tea and smoked heavily prior to this admission. Theophylline increases cAMP and diuresis. The effect of nicotine is to increase ADH and decrease diuresis, while alcohol has the opposite effect (Laurence, 1977). Therefore, smoking and tea intake should be controlled as part of treatment for water intoxication.

I am grateful to Dr D. Hailstone for his permission to report this patient.

\section{Royal Free Hospital} London NW3 2QG

\section{References}

FerrIER, I. N. (1985) Water intoxication in psychiatric illness. British Medical Journal, 291, 1594-1596.

LAURenCE, D. R. (1977) Clinical Pharmacology. London: Churchill Livingstone.

Lugtrun, S. L. \& ForsLnNG, M. (1980) Evidence for dopamine as an inhibitor of vasoprotein release in man. Clinical Endocrinology, $12,39-46$.

\section{Neuroleptic Malignant Syndrome and Compulsive Water Drinking}

SIR: The neuroleptic malignant syndrome (NMS) is thought to represent an idiosyncratic reaction to dopamine blockade, probably mediated via basal ganglia and hypothalamic pathways (Szabadi, 1984).
Compulsive water drinking has been associated with a variety of organic and psychiatric disorders, particularly psychosis (Ferrier, 1985). Although the biochemical control of thirst remains uncertain, it seems likely to involve dopaminergic pathways in the hypothalamus. Both these conditions carry a significant morbidity and mortality and are probably underdiagnosed. I report a patient who exhibited compulsive drinking while suffering NMS.

Case report: A 27-year-old woman was admitted, mute, with generalised muscle rigidity, coarse Parkinsonian tremor, sialorrhoea, difficulty swallowing, and sweating. She was indiscriminately incontinent of urine, and had episodic attacks of tachycardia and profuse sweating. She was never pyrexial. This condition had developed immediately after starting haloperidol (one week previously), and failed to respond to oral or intravenous anti-cholinergic drugs. A diagnosis of NMS was made, and all neuroleptic drugs were stopped. One week later she was observed to be drinking copious amounts of fluid; drinking from taps, water jugs, undiluted bottles of squash, and continually requesting drinks on top of those provided with her diet. She was still rigid and incontinent. The excessive drinking lasted five days, and proved impossible to quantify. Urea and electrolytes had been normal prior to excessive drinking (blood urea slightly reduced at $2.7 \mathrm{mmol} /$ litre; reference range 3.0-7.0 mmol/litre ). Two weeks after discontinuation of neuroleptic drugs her condition had completely recovered and there was no evidence of psychosis.

Although I am not aware of any reported association between excessive drinking and NMS, Wedzicha \& Hoffbrand (1984) described a case of recurrent NMS associated with hyponatraemia. The authors suggested that the hyponatraemia may have precipitated NMS, and they noted that the patient was observed to be drinking excessive water on the ward, which raises the possibility that the excessive drinking and hyponatraemia could have been secondary to NMS. The association between neuroleptic drugs and drinking behaviour is unclear. Smith \& Clarke (1980) concluded that increased thirst is likely to be related to the hyperdopaminergic state, but speculate that neuroleptic drugs may induce thirst by a mechanism similar to that proposed for tardive dyskinesia, i.e. denervation supersensitivity, occurring at hypothalamic dopamine receptors.

Once recovered, our patient was unable to recall drinking excessively or to explain it. Unfortunately, we do not have the data to comment on whether water intoxication occurred, or if there was any disturbance of antidiuretic hormone production, although this would be of great interest. It is not possible to say whether the two conditions were 
causally related, but they do have in common a disturbance of hypothalamic dopamine function and at least one reported patient with NMS (Wedzicha \& Hoffbrand, 1984) has been observed to drink excessively and to develop hyponatraemia. Given the difficulties in recognising compulsive drinking before serious consequences occur, in awareness of the possibility is essential.

\section{References}

FERRIER, I. N. (1985) Water intoxication in patients with psychiatric illness. British Medical Journal, 291, 1594.

SMITH, W. O. \& CLARKE, M. L. (1980) Self-induced water intoxication in schizophrenic patients. American Journal of Psychiatry, 137, 1055-1060.

SZABADI, E. (1984) Neuroleptic malignant syndrome. British Medical Journal, 288, 1399.

WedZicha, J. A. \& Hofrbrand, B. I. (1984) Neuroleptic malignant syndrome and hyponatraemia. The Lancet, $i, 963$.

Switch Mechanism in Afiective Illness and Oral S-Adenosylmethionine (SAM)

SIR: A mechanism for switching manic-depressives out of depression has been postulated (Bunney et al, 1970). The spontaneous switch rate from depression to hypomania or mania in untreated unipolar and bipolar depressives was reported to be $10-14 \%$ over a six-month period (Bunney, 1978; Lewis \& Winokur, 1982). Drugs acting on brain biogenic-amines, like tricyclic anti-depressants, are known to give rapid switches of mood from depression to mania; increases in these amines are thought to underlie these changes. The possibility of switching retarded, potentially suicidal depressives into a less hazardous euphoric state has long excited speculation (Bunney, 1978; Lewis \& Winokur, 1982). There would be advantages if this could be effected by means of a naturally occurring physiological brain metabolite given by a non-intimidating procedure rather than by the use of drugs or ECT.

We have recently reported (Carney et al, 1983) switching in three of 12 depressives receiving i.v. SAM. We calculated that the probability of such an event occurring by chance within six days of starting SAM was remote $(P<0.001)$ and it was probably associated with SAM. However, there was no certainty that oral SAM, unavailable during the previous study, would have the same effect as i.v. SAM.

At Northwick Park Hospital we gave SAM in daily doses of $500-1600 \mathrm{mg}$ orally for $14-42$ days in an open study to six endogenous depressive in-patients (Newcastle score 6 or more (Carney et al, 1965)).
Three became hypomanic during the trial. Thus, a 26year-old female teacher whose brother had developed mania after receiving i.v. SAM in the previous study became hypomanic. A 50 -year-old female clerk who had become hypomanic when given i.v. SAM in the former study showed a similar reaction. A 70-yearold man with no previous or family history of affective illness, unresponsive to other anti-depressant treatments, including ECT (the other two patients were considered for ECT), developed hypomania. All had been retarded, non-verbal, seclusive, and exhibited nihilistic or intrapunitive delusions. Each went through a brief euthymic period of 1-3 days before suddenly becoming elated, with marked increases in the production of speech and activity, and all developed grandiose ideas. One had heightened libido. Over the trial period the mean plasma SAM level in four of these patients increased from 30.5 to $144.0 \mathrm{ng} / \mathrm{ml}$, i.e. from 2 to 10 times the basal mean value.

We have already demonstrated that SAM can cross the blood-brain barrier and influence monoamine metabolism (Bottiglieri et al, 1984). These results with oral SAM support the earlier report of switching (Carney et al, 1983) associated with the use of i.v. SAM and again suggest that some apparently endogenous depression is SAM-responsive (Reynolds et al, 1984). SAM is the major source of methyl groups in the brain, and intimately connected with brain and amine metabolism and membrane and neuro-transmitter function. Further studies of oral SAM in endogenous depression would be worthwhile. We are currently undertaking doubleblind placebo-controlled trials of i.v. and oral SAM in patients with endogenous depression.

M. W. P. CARNEY

T. K. N. CharY

T. BOTTIGLIERI

Northwick Park Hospital \&

Clinical Research Centre

Harrow, Middx

E. H. REYNOLDS

B. K. TOONE

Kings College Hospital

Denmark Hill, London SES

\section{Reforence:}

Bottiglierl, T., Laundy, M., Martin, R., Carney, M. W. P., Nissenbaum, H., TOONE, B. K., Johnson, A. L. \& ReYnolds, E. H. (1984) S-adenosylmethionine influences monoamine metabolism. The Lancet, ii, 224.

BUNNEY, W. F. (1978) Psychopharmacology of the switch process in afiective disorder. In Psychopharmacology: A Generation of Progress (eds M. A. Lipton, A. Damascio \& K. F. Kellanil). New York: Raven Press. 